\title{
Validation of a Standardized Normalization Template for Statistical Parametric Mapping Analysis of ${ }^{123}$ I-FP-CIT Images
}

\author{
Aurélie Kas ${ }^{1,2}$, Pierre Payoux ${ }^{3}$, Marie-Odile Habert ${ }^{2,4}$, Zoulikha Malek ${ }^{2}$, Yann Cointepas ${ }^{5}$, Georges El Fakhri ${ }^{6}$, \\ Philippe Chaumet-Riffaud ${ }^{7}$, Emmanuel Itti $^{8}$, and Philippe Remy ${ }^{1,9}$ \\ ${ }^{1}$ URA CNRS-CEA 2210, Service Hospitalier Frédéric Joliot, Orsay, France; ${ }^{2}$ Service de Médecine Nucléaire, CHU Pitié-Salpêtrière, \\ AP-HP, Paris, France; ${ }^{3}$ Service de Médecine Nucléaire, CHU Purpan et INSERM U825, Toulouse, France; ${ }^{4}$ Université Pierre et Marie \\ Curie-Paris 6, INSERM U678, Paris, France; ${ }^{5}$ UNAF-CEA, Service Hospitalier Frédéric Joliot, Orsay, France; ${ }^{6}$ Nuclear Medicine \\ Division, Radiology Department, Harvard Medical School and Brigham and Women's Hospital Boston, Massachusetts; ${ }^{7}$ Service de \\ Médecine Nucléaire, CHU de Bicêtre, AP-HP et Faculté de Médecine Paris 11, Le Kremlin Bicêtre, France; ${ }^{8}$ Service de Médecine \\ Nucléaire, CHU Henri Mondor, AP-HP et Faculté de Médecine Paris 12, Créteil, France; and ${ }^{9}$ Département de Neurosciences, \\ CHU Henri Mondor, AP-HP et Faculté de Médecine Paris 12, Créteil, France
}

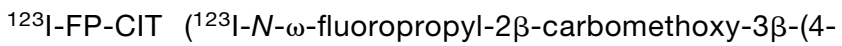
iodophenyl)nortropane) is a SPECT dopamine transporter (DAT) tracer that probes dopaminergic cell loss in Parkinson's disease (PD). Quantification of ${ }^{123}$ I-FP-CIT images is performed at equilibrium using a ratio (BR) of specific (striatal) to nonspecific (occipital) uptake with values obtained from regions of interest drawn manually over these structures. Statistical parametric mapping (SPM) is a fully automated voxel-based statistical approach that has great potential in the context of DAT imaging. However, the accuracy of the spatial normalization provided by SPM has not been validated for ${ }^{123}$-FP-CIT images. Our first aim was to create an ${ }^{123}$ I-FP-CIT template that does not require the acquisition of patient-specific MRI and to validate the spatial normalization procedure. Next, we hypothesized that this customized template could be used by different SPECT centers without affecting the outcomes of imaging analyses. Methods: The spatial normalization to the customized template created with SPM (template A1) was validated using ${ }^{123}$ I-FP-CIT images obtained from 6 subjects with essential tremor (ET) with normal DAT status and 6 PD patients. Variability in BR values due to the normalization was evaluated using striatal volume of interest (VOI). To determine whether different SPECT centers could use a unique ${ }^{123}$-FP-CIT template, we generated 3 other ${ }^{123} \mathrm{I}-\mathrm{FP}-\mathrm{CIT}$ templates using different subjects and image-processing schemes. The interchangeability of these templates was assessed using (a) putamen BR values analyzed with the intraclass correlation coefficient (ICC) and the Bland-Altman graphical analysis, and (b) SPM analysis comparing the results of group comparisons - that is, ET versus PD, obtained after normalization to each of the 4 templates. Results: There was no significant difference between pre- and postnormalization striatal BR values in our study. The mean variability calculated with putamen VOI values after normalization to each template was $<10 \%$, with the lowest ICC of $98 \%$. Intergroup analyses per-
\end{abstract}

Received Dec. 8, 2006; revision accepted Jun. 14, 2007.

For correspondence or reprints contact: Aurélie Kas, MD, URA CNRS-CEA 2210, Service Hospitalier Frédéric Joliot, 4, place du Général Leclerc, 91401 Orsay, France.

E-mail: aurelie.kas@psl.aphp.fr

COPYRIGHT @ 2007 by the Society of Nuclear Medicine, Inc. formed with VOI and SPM approaches provided similar results independently of the template used. Conclusion: SPM normalization was accurate even in subjects with low striatal ${ }^{123}$ FP-CIT uptake, making it a promising approach for automatic analysis of ${ }^{123}$-FP-CIT images using a single customized template at different centers.

Key Words: dopamine transporters; SPECT; statistical parametric mapping; normalization; Parkinson's disease

J Nucl Med 2007; 48:1459-1467

DOI: 10.2967/jnumed.106.038646

$\mathbf{P}$ arkinson's disease (PD) is characterized by the progressive degeneration of nigrostriatal dopaminergic neurons. This neurodegenerative process is associated with a loss of striatal dopamine transporters (DATs) as shown by postmortem studies $(1,2)$. Therefore, in vivo measurement of DAT density with PET or SPECT is an early marker of the dopaminergic cell loss in subjects with parkinsonian symptoms or in asymptomatic carriers of genetic mutations causing PD (3-7). In clinical routine, DAT SPECT images are often analyzed visually. However, quantitative analysis is useful to differentiate subjects with subtle localized or diffuse loss of DATs that can be difficult to sort out by visual inspection alone. Moreover, quantification is mandatory to measure disease progression $(7-11)$ and to assess the efficacy of neuroprotective drugs $(12,13)$.

DAT availability can be estimated using a ratio between specific (striatal) to nonspecific (e.g., occipital) activity $(14,15)$ measured using regions of interest (ROIs) drawn manually over these structures in individual images. However, manual ROI delineation is operator-dependent and may be affected by the variability in head positioning and severe signal loss that occurs in the posterior putamen of PD patients. Therefore, manual drawing of ROIs is associated 
with an intraoperator and an interoperator variability, hampering the comparison of images from different centers. To overcome such limitations, DAT SPECT images could be analyzed using automated voxel-based statistical methods, such as statistical parametric mapping (SPM; Wellcome Department of Cognitive Neurology, London, U.K.) $(16,17)$, which has been shown to be more sensitive than ROI analysis (18).

To perform SPM comparisons, a "spatial normalization" of individual images is required. Spatial normalization consists of applying linear and nonlinear transformations to register every brain volume to a standardized brain template within the Montreal Neurological Institute neuroanatomic space (MNI; http://www.bic.mni.mcgill.ca). The normalization algorithm uses a matching criterion based on minimizing the sum of squared differences between image and template voxel values (19). For this criterion to be successful, the individual image being normalized and the template must have a similar intensity profile $(20,21)$. Therefore, DAT SPECT images cannot be normalized using cerebral blood flow (CBF) or glucose metabolism templates provided within the SPM software package. The use of SPM for DAT images requires the creation of a dedicated template and the validation of the accuracy of image transformations as well as the conservation of structure intensity during spatial normalization, especially for pathologic ${ }^{123} \mathrm{I}-\mathrm{FP}-\mathrm{CIT}\left({ }^{123} \mathrm{I}-\mathrm{N}-\omega\right.$ fluoropropyl-2 $\beta$-carbomethoxy-3 $\beta$-(4-iodophenyl)nortropane) images. Indeed, image artifacts have been reported after spatial normalization of brain images with focal lesions, where the normalization algorithm erroneously attempted to reduce mismatch between template and image intensities at the site of the abnormalities (22).

Our first aim was to create and validate a dedicated template for ${ }^{123}$ I-FP-CIT that does not require the acquisi- tion of individual MRI for the spatial normalization procedure. We constructed a "house-made" template based on a set of normal DAT images obtained in a single center. The validation of the normalization process was performed using a test sample of 6 PD patients and 6 subjects with essential tremor (ET).

Our second objective was to evaluate whether each center should create its own template to normalize DAT images acquired locally, taking into account the characteristics of its SPECT camera and the data-processing schemes, or whether it is feasible to use a unique template across different centers. To verify this assumption, we created 3 other templates from normal ${ }^{123}$ I-FP-CIT images obtained in other centers. We evaluated the impact of each normalization procedure on the results of the comparison between the same $6 \mathrm{ET}$ and 6 PD patients with both ROI and SPM analyses. If our hypothesis is confirmed, a customized template could be made available to the scientific community to encourage a widespread use of SPM to analyze ${ }^{123}$ I-FP-CIT images in research as well as in the clinical setting.

\section{MATERIALS AND METHODS}

\section{Subjects}

Two categories of subjects were selected in this study: First, a total of 30 subjects-25 with ET and 5 healthy volunteers-all having a normal ${ }^{123}$ I-FP-CIT image, were used for the construction of the different templates (Table 1). Next, a "test population" was selected in Toulouse, France, to validate the normalization process and the comparison between the different templates. This test population consisted in 6 subjects with a clinically diagnosed ET (mean age $\pm \mathrm{SD}=64.2 \pm 10.8 \mathrm{y} ; 3$ men, 3 women) and 6 patients (mean age $\pm \mathrm{SD}=58.3 \pm 9.9 \mathrm{y} ; 3$ men, 3 women) with PD predominating in the left hemibody. Clinical diagnosis of PD was based on the U.K. Parkinson's Disease Society Brain Bank

TABLE 1

Characteristics of Image Acquisition and Data Processing Used to Create 4 Templates

\begin{tabular}{|c|c|c|c|c|}
\hline Template & A1 & A2 & $\mathrm{B}$ & $\mathrm{C}$ \\
\hline Center & \multicolumn{2}{|c|}{ Toulouse, France } & Créteil, France & Paris, France \\
\hline Camera & \multicolumn{2}{|c|}{ Triple-head IRIX 3 (Picker) } & Dual-head AXIS (Philips) & Triple-head IRIX 3 (Picker) \\
\hline Collimators & \multicolumn{2}{|c|}{ LE-HR parallel } & LE-HR parallel & LE-HR parallel \\
\hline Controls & \multicolumn{2}{|c|}{ ET } & ET & Healthy volunteers \\
\hline No. of controls & \multicolumn{2}{|c|}{15} & 10 & 5 \\
\hline Acquisition parameters & \multicolumn{2}{|c|}{120 projections $/ 360^{\circ}, 128^{2}$} & 120 projections $/ 360^{\circ}, 128^{2}$ & 120 projections $/ 360^{\circ}, 128^{2}$ \\
\hline $\begin{array}{l}\text { Reconstruction parameters } \\
\text { (iteration, subsets) }\end{array}$ & OSEM (12-6) & OSEM (6-4) & OSEM (4) & OSEM (12-6) \\
\hline Software/workstation & Hermes & Segami-Mirage & Odyssey & Hermes \\
\hline $\begin{array}{l}\text { Homogeneous attenuation } \\
\text { correction }\end{array}$ & $\mu=0.15$ & No & $\mu=0.15$ & $\mu=0.15$ \\
\hline Scatter correction & No & No & No & Yes \\
\hline $\begin{array}{l}\text { Variable collimator response } \\
\text { correction }\end{array}$ & No & No & No & Yes \\
\hline $\begin{array}{l}\text { 3D postfiltering } \\
\text { (order, cut-off frequency) }\end{array}$ & $\begin{array}{l}\text { Butterworth, 5, } 1.7 \\
\text { cycles } \cdot \mathrm{cm}^{-1}\end{array}$ & Wiener & $\begin{array}{l}\text { Butterworth, } 4,0.35 \\
\text { cycle/pixel }\end{array}$ & $\begin{array}{l}\text { Butterworth, 5, } 1.7 \\
\text { cycles } \cdot \mathrm{cm}^{-1}\end{array}$ \\
\hline $\begin{array}{l}\text { LE-HR = low energy, high re } \\
\text { dimensional. }\end{array}$ & tion; OSEM = orc & d-subsets exp & ion maximization; $\mu=$ at & ation coefficient $\left(\mathrm{cm}^{-1}\right) ; 3 \mathrm{D}$ \\
\hline
\end{tabular}


criteria (23). The mean duration of PD at the time of SPECT assessment was $4.5 \mathrm{y}$ (range, 1-8 y). The severity of PD was assessed using the Hoehn-Yahr scale and the motor score of the Unified Parkinson's Disease Rating Scale (UPDRS-3) measured $12 \mathrm{~h}$ after withdrawal of antiparkinsonian medication (24). The mean UPDRS-3 score and Hoehn-Yahr stage were 26.0 \pm 18.6 and $2.6 \pm 0.7$, respectively. Two patients had an early PD with a Hoehn-Yahr stage of $<2$ and a UPDRS-3 score of $<10$. All subjects provided informed consent before participating in this study.

\section{SPECT Acquisition and Data Processing}

All subjects received potassium perchlorate to block thyroid uptake. Images were acquired $3 \mathrm{~h}$ after intravenous injection of 150-170 MBq of ${ }^{123}$ I-FP-CIT (DaTSCAN; GE Healthcare) (25). The characteristics of the $\gamma$-cameras used in the different centers and the image reconstruction algorithms are listed in Table 1.

The test set of twelve ${ }^{123}$ I-FP-CIT images was acquired in Toulouse, on a triple-head $\gamma$-camera equipped with an ultra-highresolution and low-energy parallel collimator (Table 1). The 120 projections were reconstructed using an iterative algorithm (HOSEM; 12 iterations, 6 subsets; HERMES Nuclear Diagnostics AB, Sweden) that modeled uniform attenuation in the projector/backprojector. The reconstructed volume (voxel size $=2.3 \times 2.3 \times 2.3 \mathrm{~mm}^{3}$ ) was filtered using a Butterworth filter (order, 5; cutoff frequency, 1.7 cycle $\cdot \mathrm{cm}^{-1}$ ).

All images were converted from the Interfile to the Analyze format (Xmedcon software; http://xmedcon.sourceforge.net/). Quantification of the DAT-ligand binding was based on the specific-to-nonspecific binding ratio (BR) measured at equilibrium $(14,15)$. In each brain voxel, the specific uptake was divided by the nonspecific uptake measured with an $11.4 \mathrm{~mL}$ volume of interest (VOI) positioned over the occipital cortex (26) to obtain parametric images of BR (BrainVisa software; http://brainvisa.info).

\section{Creation of Reference ${ }^{123}$ I-FP-CIT Template: A1}

Template A1 was created with normal parametric images of 15 subjects with ET (age, $67 \pm 11.5 \mathrm{y}$ ). These images were obtained in the same center and with the same acquisition and reconstruction procedures as the test population (Table 1).

All processing was performed using SPM under Matlab (Mathworks Inc.). We have deliberately chosen the SPM99 version, rather than SPM2 version, because the normalization process in the latter, in contrast with the former, yielded several misregistrations. To place the 15 parametric images in the standard MNI space, a MNI-based template of ${ }^{11} \mathrm{C}$-raclopride was used because of its high specific striatal binding and low cortical uptake, which is similar to the profile of ${ }^{123} \mathrm{I}$-FP-CIT images. To register ${ }^{123} \mathrm{I}$-FPCIT images with this template, we used the normalization algorithm provided by SPM that involves a 12-parameter affine transformation followed by nonlinear deformations. Basis functions for the nonlinear warping were $7 \times 8 \times 7$ in $x$-, $y$-, and $z$-dimensions, respectively, with 12 iterations. Bilinear interpolation was used during final reslicing. The dimensions of output volumes were $x=-90: 91, y=-126: 91$, and $z=-72: 109 \mathrm{~mm}$ from the anterior commissure, with a $2 \times 2 \times 2 \mathrm{~mm}^{3}$ isotropic voxel. Finally, the ${ }^{123}$ I-FP-CIT template was obtained by averaging the normalized images and their symmetric (mirror) image and filtering using a 3-dimensional (3D) gaussian kernel (full width at half maximum $[\mathrm{FWHM}]=8 \mathrm{~mm}$ ) (Figs. 1 and 2).
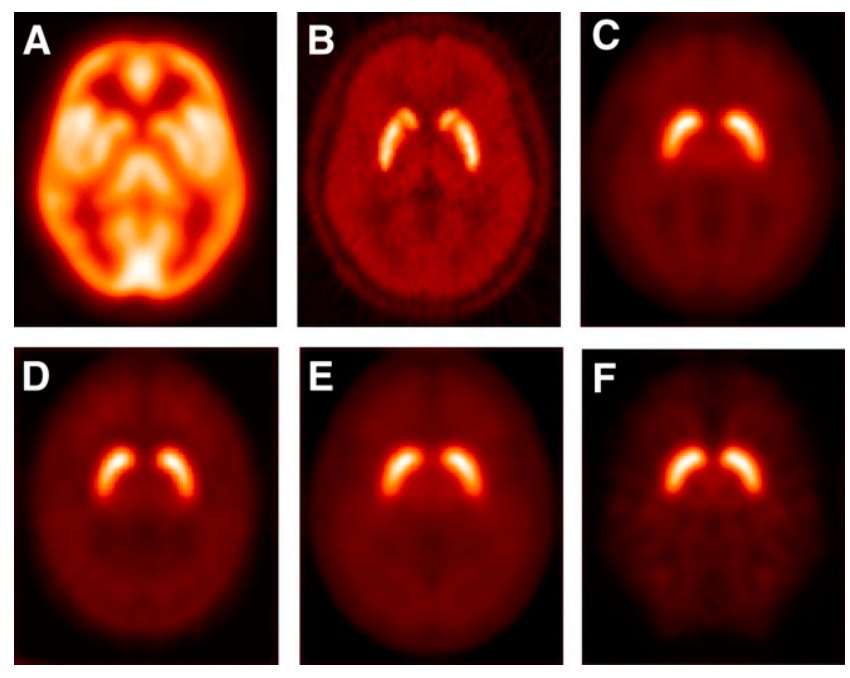

FIGURE 1. Templates: (Top) Regional CBF template (A) available in SPM software package; ${ }^{11} \mathrm{C}$-raclopride template (B) created in Orsay PET Center from images of healthy subjects; reference template of ${ }^{123} \mathrm{I}-\mathrm{FP}-\mathrm{CIT}$ (C) created in center A (Template A1). Raclopride template has high specific striatal and low cortical uptake similar to the profile of ${ }^{123} \mathrm{I}-\mathrm{FP}-\mathrm{CIT}$ images. (Bottom) Three other templates of ${ }^{123} \mathrm{I}-\mathrm{FP}-\mathrm{CIT}(\mathrm{D}=$ template $\mathrm{A} 2, \mathrm{E}=$ template $\mathrm{B}$, and $\mathrm{F}=$ template $\mathrm{C}$ ) constructed with images obtained from different $\gamma$-cameras or data-processing schemes.

\section{Creation of 3 Additional Normalization Templates}

To test the assumption that DAT images acquired onsite could be normalized to an ${ }^{123}$ I-FP-CIT template generated by another center, 3 additional templates were created using the same SPM procedure as for template A1 and tested on the same test population.

Given the fact that the $\gamma$-cameras in the 3 centers were only slightly different, these cameras were calibrated before the beginning of the study using a Radiology Support Devices (RSD) phantom to determine the point spread function (PSF). The PSF was different between the 3 systems, suggesting that there was center effect. In addition, to increase the variability between the templates, each center used its own set of normal images acquired locally and its usual acquisition protocol and data-processing scheme (Table 1).

Template A2 was created using the same set of acquired projections as template A1 but images were not corrected for attenuation and were filtered post hoc with a Wiener filter. Template B was computed with images obtained in 10 ET subjects (mean age, $64.4 \pm 12.5$ y) scanned with a dual-head camera. Template $C$ was created using images obtained in 5 healthy volunteers (mean age $=$ $58.8 \pm 16$ y) acquired with a triple-head camera. In this center, projections were acquired in 8 energy windows ranging from 79 to $179 \mathrm{keV}$, corrected for scatter using a validated artificial neural network (27) and reconstructed with an iterative algorithm that modeled attenuation and variable collimator response in the projector/backprojector of an OSEM algorithm (Fig. 1).

\section{Spatial Normalization of Test Population}

The 12 parametric images (6 ET and $6 \mathrm{PD}$ ) were spatially normalized to each template under identical conditions, including the 12-parameters' affine transformation, $7 \times 8 \times 7$ discrete cosine transform basis functions, and 12 iterations of nonlinear optimization. 

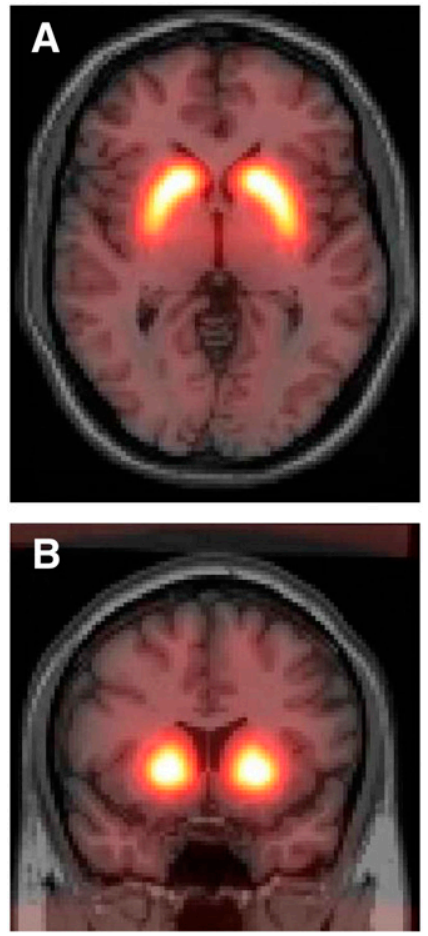

FIGURE 2. Template A1 overlaid on single-subject brain MRI available in SPM in 3 orthogonal views: $A$, axial; $\mathrm{B}$, coronal; and $\mathrm{C}$, sagittal. Normalized striatal 123-FP-CIT binding is accurately superimposed onto striatum.

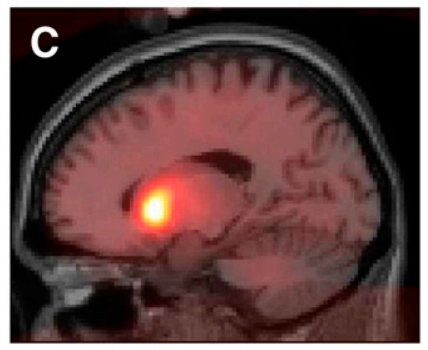

The voxel size was set to $2 \times 2 \times 2 \mathrm{~mm}^{3}$. The normalized images were spatially smoothed with a gaussian kernel of 10 -mm full width at half maximum (FWHM) to accommodate interindividual anatomic variability and improve the signal-to-noise ratio (28).

Impact of Spatial Normalization to Template A1 on ${ }^{123}$ FP-CIT Quantification

The impact of nonlinear transformations induced by the spatial normalization on the striatal BR values was determined using the test set of normal (ET) and abnormal (PD) images. The BR values were measured bilaterally in the striatum of each subject before and after spatial normalization to the A1 template. In all subjects, the transaxial slices of native and normalized parametric images, containing the specific activity within the striatum, were summed, including 1 slice extending beyond visually identified striatum. Standardized, circular ROIs $\left(552 \mathrm{~cm}^{2}\right)$ were positioned using the Anatomist software (http://brainvisa.info) in the summed slices over the caudate nucleus and putamen, both in native and normalized images. The differences in BR values obtained in the left and right striatum before and after normalization were compared using a paired $t$ test. In addition, a comparison between PD and ET subjects was performed using values obtained before and after normalization and a Student $t$ test, to ensure that the difference between the 2 groups was preserved despite the spatial normalization. For these analyses, the right and the left striatum were considered as independent variables ( 24 values).

\section{Validation of Use of a Template Obtained in a Different SPECT Center}

We performed 2 approaches to investigate the interchangeability of the 4 templates. First, we compared the BR values in the test population after normalization to the templates $\mathrm{A} 2, \mathrm{~B}$, and $\mathrm{C}$ with the BR values obtained after normalization to the reference template A1. This was done by calculating the percentage of variability between each normalization procedure and using the Bland-Altman graphical analysis and the intraclass correlation coefficient (ICC). Next, we determined the influence of each normalization template on the results of the comparison between PD and ET subjects performed using both VOI and SPM analyses. The 2 approaches are detailed below.

Comparison of Normalized VOI Values Across 4 Templates. The outcome value was the BR measured with VOI in both putamen (pBR) on normalized images. The putamen was chosen because it is the striatal region that is the most affected in PD (29) and, therefore, where normalization is more likely to fail. To assess the same region in all subjects after the 4 different normalizations, VOIs were delineated in 3D using the Anatomist software on the putamen of the high-resolution T1-weighted MRI from the MNI and then transferred automatically onto each individual normalized image. The right and the left putamen were considered as independent variables.

The variability between the reference template (A1) and the A2 template was calculated as follows:

$$
\frac{\left|\mathrm{pBR}_{\mathrm{A} 1}-\mathrm{pBR}_{\mathrm{A} 2}\right|}{\left(\mathrm{pBR}_{\mathrm{A} 1}+\mathrm{pBR}_{\mathrm{A} 2}\right) / 2} \times 100 \% \text {. }
$$

Eq. 1

The reliability was estimated by the ICC and $95 \%$ limits of agreement (30). To calculate the ICC, repeated-measures ANOVA was performed to obtain variance between and within subjects.

The ICC was expressed as:

$$
\mathrm{ICC}=\frac{\text { MSBS }- \text { MSWS }}{\mathrm{MSBS}+(\mathrm{k}-1) \mathrm{MSWS}}
$$

where MSBS and MSWS are the mean sum of squares between and within subjects, respectively, and $\mathrm{k}$ is the number of withinsubjects measurements. The $95 \%$ limits of agreement were determined by the Bland-Altman graphical analysis: The difference in $\mathrm{pBR}$ values between the $\mathrm{A} 1$ and the $\mathrm{A} 2$ normalizations $\left(\mathrm{pBR}_{\mathrm{A} 1}-\right.$ $\left.\mathrm{pBR}_{\mathrm{A} 2}\right)$ were plotted against their mean $\left(\mathrm{pBR}_{\mathrm{A} 1}+\mathrm{pBR} \mathrm{A}_{\mathrm{A} 2}\right) / 2$ for both putamen in each patient. We examined the agreement between the 2 measurements by computing the spread of the difference scores around the center line representing 0 difference (31).

The same analysis was repeated for the templates B and C compared with A1.

Impact of Change of Template on Differences Between PD and ET Subjects. We determined the influence of the normalization template on the statistical outcomes of the comparison between PD and ET subjects. This was performed for each of the 4 normalization processes using 2 approaches: (a) with the BR values measured in right and left putamen using the VOI and a Student $t$ test and (b) with SPM. This voxelwise analysis comparing ET and PD subjects was performed with an analysis of covariance (ANCOVA) with age as a covariable. The height 
threshold was set at $P_{\text {uncorrected }}<0.001$ with a minimum cluster size of 20 voxels $\left(160 \mathrm{~mm}^{3}\right)$. A mask, including the midbrain and basal ganglia, was applied to improve statistical power (smallvolume correction). A SPM $t$ map was obtained for each template. The A2, B, and C SPM $t$ maps were compared with the reference SPM $t$ map A1. We analyzed (a) the volume and peak coordinates of the regions that were statistically different between the groups ("clusters") and (b) the percentage of each statistical cluster belonging to the different structures of the striatum using the Anatomic Automatic Labeling software (AAL; http://www.cyceron. fr/freeware) (32).

\section{RESULTS}

\section{Impact of Spatial Normalization to Template A1 on 123I-FP-CIT Quantification}

There was no significant difference of BR values in the left and right striatum before and after normalization to template A1 $(n=24, P=0.08,95 \%$ confidence interval $[\mathrm{CI}]=[-0.04,0.03])$. Striatal BR values were significantly decreased in the PD compared with the ET patients before normalization: $-52.9 \%(t=-7.0, P<0.0001)$ and $-45.3 \%$ ( $t=-5.6, P=0.0002$ ) for the right and left striatum, respectively. The same comparison performed after normalization provided similar results: $-53.2 \%(t=-7.0, P<$ $0.0001)$ and $-45.2 \%(t=-5.7, P=0.0002)$ for the right and left striatum, respectively.

\section{Validation of Use of a Template Obtained in a Different SPECT Center}

Comparison of Normalized VOI Values Across 4 Templates. The BR values in the putamen after normalization to templates $\mathrm{A} 2, \mathrm{~B}$, and $\mathrm{C}$ were highly reproducible when compared with the values obtained after normalization to template A1, with a mean variability of $<10 \%$ and a high correlation with a lowest ICC equal to $98 \%$ (Table 2; Fig. 3 ).

Impact of Change of Template on Differences Between $P D$ and ET Subjects. Both VOI and SPM analyses showed that the comparison between ET and PD patients provided similar results regardless of which template was used for normalization.

\section{VOI Analysis}

BR values were significantly decreased in PD patients compared with ET patients after normalization to template A1: $-56.3 \%(t=-9.7, P<0.0001)$ and $-42.9 \%(t=$ $-5.8, P=0.0002$ ) for the right and left putamen, respec-

TABLE 2

Evaluation of Reproducibility of BR Values Obtained from Normalization Procedure Across Templates

\begin{tabular}{cccc}
\hline Template & $\begin{array}{c}\text { Variability in \% } \\
\text { (mean } \pm \text { SD) }\end{array}$ & $\begin{array}{c}\text { ICC } \\
{[95 \% \mathrm{Cl}]}\end{array}$ & $\begin{array}{c}\text { 95\% limits of } \\
\text { agreement }\end{array}$ \\
\hline A2 vs. A1 & $3.69 \pm 3.04$ & $0.99[0.98-0.99]$ & -0.11 to 0.24 \\
B vs. A1 & $4.45 \pm 3.10$ & $0.99[0.98-0.99]$ & -0.13 to 0.23 \\
C vs. A1 & $7.01 \pm 3.75$ & $0.98[0.96-0.99]$ & -0.02 to 0.28 \\
\hline
\end{tabular}
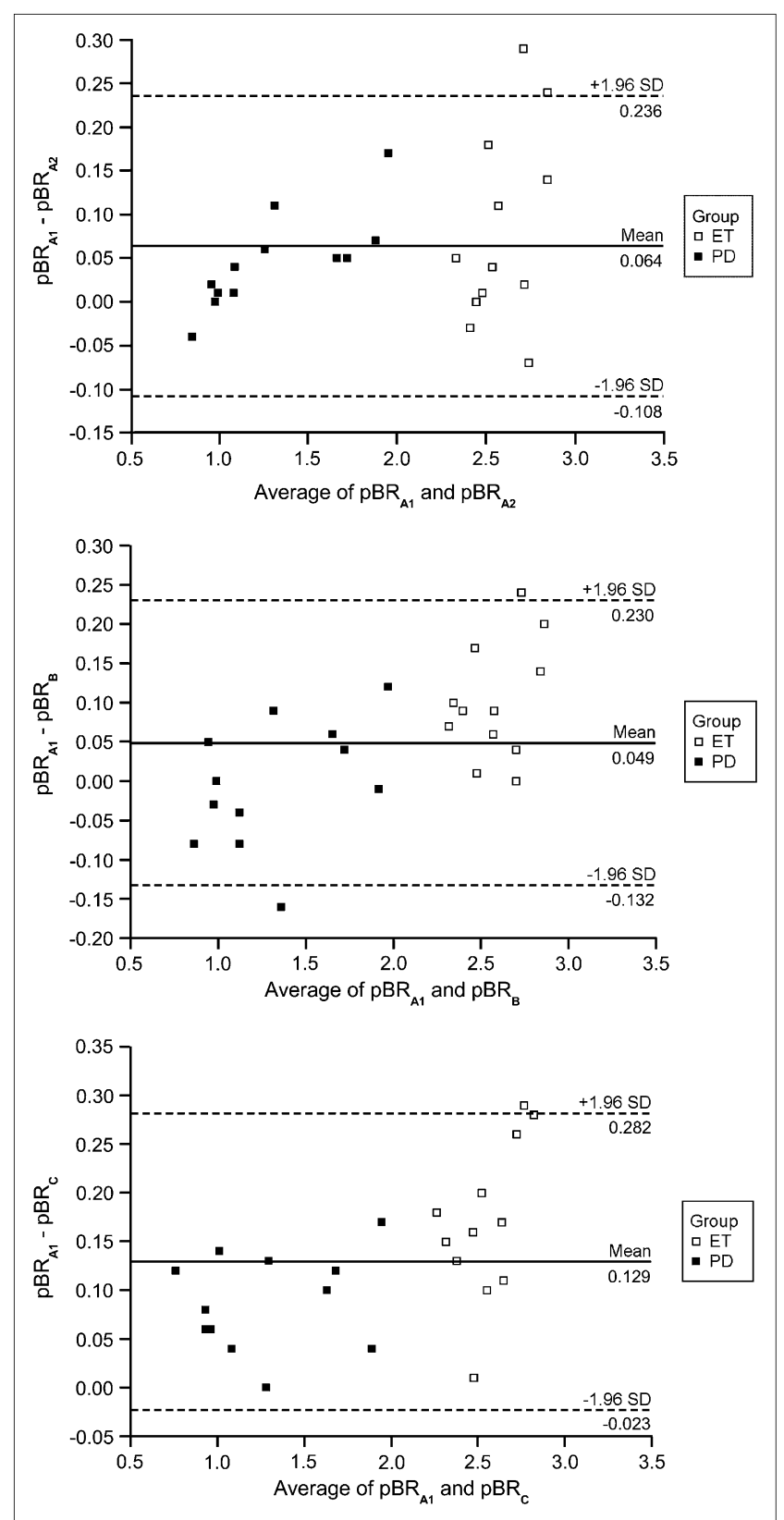

FIGURE 3. Difference against average of putaminal ratios (pBR) obtained with template A1 and 3 other templates, with 95\% limits of agreement. Solid line shows mean difference score. The $95 \%$ limits of agreement (dashed lines) represent 1.96 SDs above and below the mean difference score. Each graph compares the reproducibility between the method using templates $\mathrm{A} 2, \mathrm{~B}$, and $\mathrm{C}$ and template $\mathrm{A} 1$.

tively. The same comparison performed after the normalizations to the other templates provided a difference having a similar magnitude and significance (Fig. 4) template A2: $-55.7 \%(t=-10.3, P<0.0001)$ and $-43.9 \%(t=-6.4$, $P<0.0001)$ for the right and left putamen, respectively; template B: $-53.3 \%(t=-9.0, P<0.0001)$ and $-41.5 \%$ $(t=-6.0, P=0.0001)$; template $\mathrm{C}:-56.1 \%(t=-9.3$, $P<0.0001)$ and $-43.2 \%(t=-5.6, P=0.0002)$. 


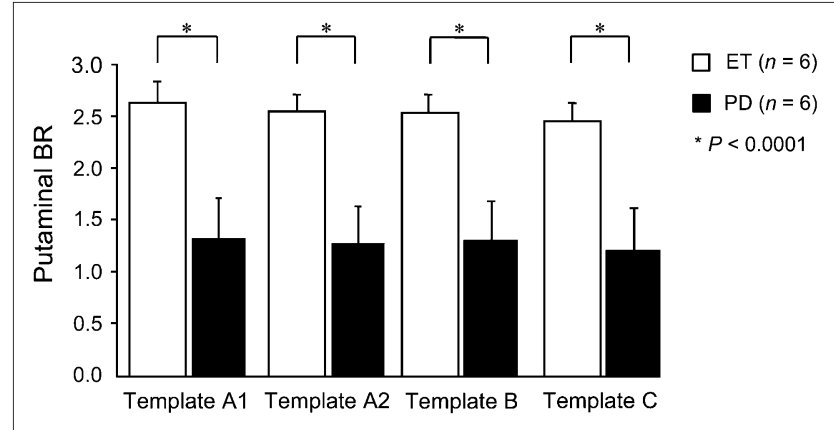

FIGURE 4. Mean $\mathrm{BR} \pm \mathrm{SD}$ values measured with VOI in putamen of subjects with ET ( $\square$ ) and PD ( $\square$ ), after each normalization. Right and left putamen values have been averaged. Mean decrease in PD group compared with ET group was $-49.6 \%$, $-49.8 \%,-47.4 \%$, and $-49.7 \%$ for templates $A 1, A 2, B$, and $C$, respectively (Student $t$ test, all $P<0.0001$ ).

\section{SPM Analysis}

The comparison between ET and PD subjects yielded 4 SPM $t$ maps according to the different templates (Fig. 5). Significant differences were observed bilaterally in the striatum, with a more severe decrease in the right putamen, which was the most affected side in the PD patients. The topography, the volume of the clusters, and the $Z$ scores were similar after analyses performed with the different templates (Table 3). In addition, the analysis performed with the AAL software showed similar repartition of significant voxels in the caudate and putamen independently of the normalization template procedure (Table 4).

\section{DISCUSSION}

In this pilot study, we created 4 different templates to normalize ${ }^{123}$ I-FP-CIT SPECT images. First, we found no difference in ${ }^{123}$ I-FP-CIT quantification before and after normalization in a sample of ET and PD patients. Moreover, the choice of the SPM template has little effect on the results of the comparison between PD and ET subjects. Although, the templates were different, the 4 normalization procedures provided results close enough to consider that the templates can be used interchangeably without altering the clinical interpretation of the comparison. This suggests that a single template of ${ }^{123}$ I-FP-CIT may be used to normalize DAT independently of the acquisition and reconstruction protocols used in nuclear medicine centers.

Spatial normalization, which is a crucial step in the voxel-based analysis, required validation for ${ }^{123}$ I-FP-CIT images. Indeed, normalization can be performed on the basis of the MRI of individual subjects using the MRI template provided within the SPM software $(19,20,33)$. This strategy is the most accurate because of the high spatial resolution of anatomic images (20) but requires the acquisition of an MRI scan for each individual undergoing DAT SPECT. Moreover, it requires an accurate registration of the DAT image onto the individual MR image. These constraints make such an approach difficult to implement in clinical routine. An alternative approach consists of performing a normalization based solely on functional images using the CBF template available in SPM $(21,34)$. However, because the normalization algorithm minimizes the

FIGURE 5. SPM $t$ maps $\left(P_{\text {uncorrected }}<\right.$ $0.001)$ obtained by comparing ET patients with PD patients ( $6 \mathrm{PD}<6 \mathrm{ET})$ after normalization to each template. Four statistical maps reveal similar significant differences between the 2 groups for 4 different templates. $R=$ right; $L=$ left.

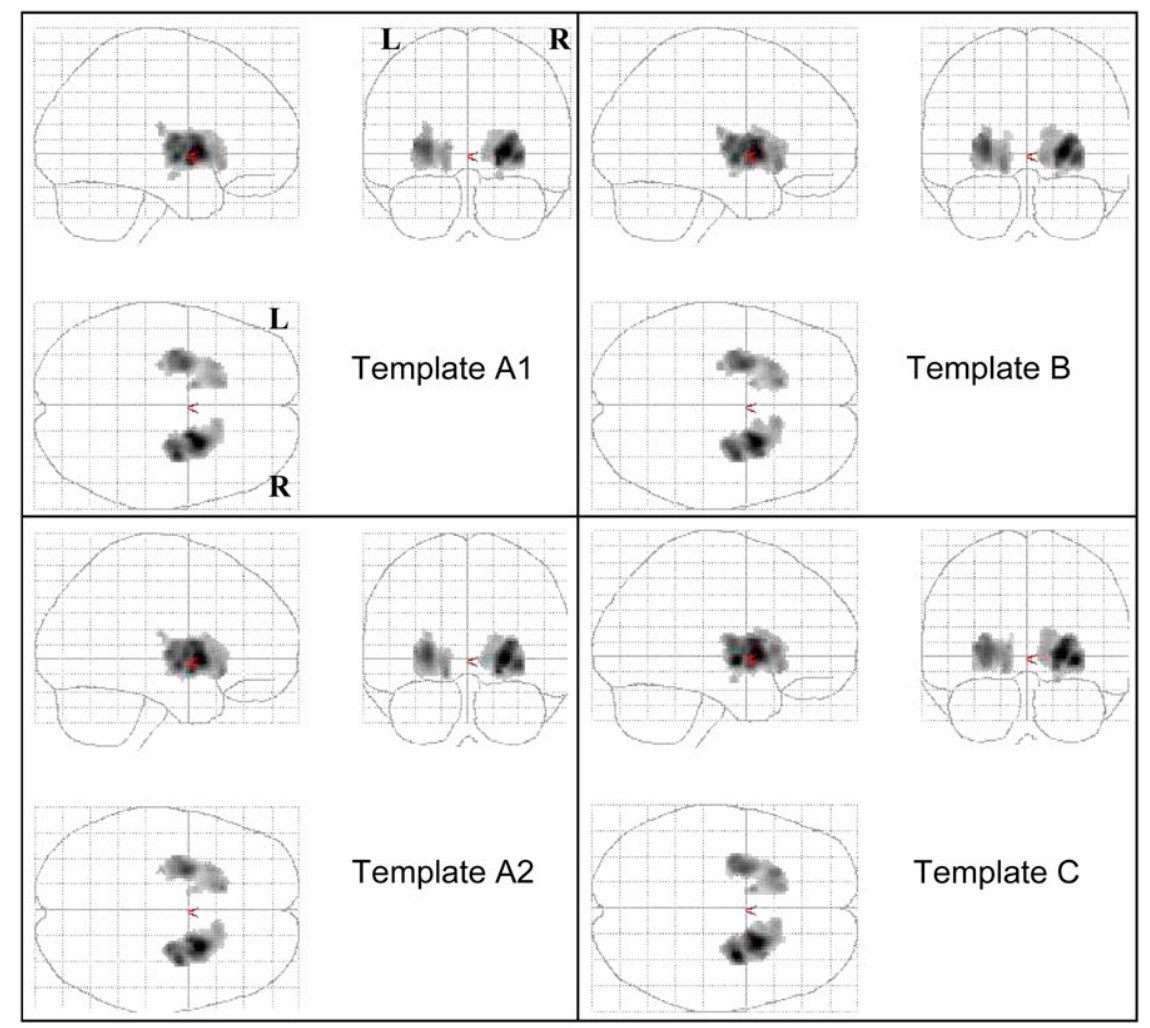


TABLE 3

SPM Results in ET Subjects Compared with PD Subjects According to Template Used for Normalization

\begin{tabular}{|c|c|c|c|c|c|c|c|c|c|c|}
\hline \multicolumn{3}{|c|}{ Cluster level } & \multicolumn{3}{|c|}{ Voxel level } & \multicolumn{4}{|c|}{ MNI coordinates (mm) } & \multirow[b]{2}{*}{ Location } \\
\hline$P_{\text {corrected }}$ & $\mathrm{K}_{\mathrm{E}}$ & $P_{\text {uncorrected }}$ & $P_{\text {corrected }}$ & $Z$ score & $P_{\text {uncorrected }}$ & $t$ & $x$ & $y$ & $z$ & \\
\hline \multicolumn{11}{|c|}{ ET $>$ PD with template A1 } \\
\hline \multirow[t]{3}{*}{0.000} & 1,415 & 0.000 & 0.001 & 5.30 & 0.000 & 14.97 & 24 & 6 & 2 & R putamen \\
\hline & & & 0.004 & 4.96 & 0.000 & 12.13 & 32 & -2 & -8 & \\
\hline & & & 0.009 & 4.79 & 0.000 & 10.94 & 28 & -8 & 10 & \\
\hline \multirow[t]{3}{*}{0.000} & 1,074 & 0.000 & 0.014 & 4.71 & 0.000 & 10.45 & -28 & -2 & 4 & L putamen \\
\hline & & & 0.024 & 4.60 & 0.000 & 9.79 & -34 & -8 & 6 & \\
\hline & & & 0.164 & 4.06 & 0.000 & 7.23 & -14 & 4 & 4 & \\
\hline \multicolumn{11}{|c|}{ ET $>$ PD with template A2 } \\
\hline \multirow[t]{3}{*}{0.000} & 1,506 & 0.000 & 0.000 & 5.40 & 0.000 & 15.91 & 24 & 8 & 0 & R putamen \\
\hline & & & 0.003 & 5.02 & 0.000 & 12.59 & 20 & 2 & -8 & \\
\hline & & & 0.003 & 5.01 & 0.000 & 12.50 & 28 & 4 & 10 & \\
\hline \multirow[t]{3}{*}{0.000} & 1,095 & 0.000 & 0.005 & 4.90 & 0.000 & 11.70 & -26 & -2 & 2 & L putamen \\
\hline & & & 0.057 & 4.40 & 0.000 & 8.74 & -14 & 22 & $-\overline{8}$ & \\
\hline & & & 0.154 & 4.06 & 0.000 & 7.22 & -26 & 16 & 4 & \\
\hline \multicolumn{11}{|c|}{ ET $>$ PD with template B } \\
\hline \multirow[t]{3}{*}{0.000} & 1,478 & 0.000 & 0.000 & 5.39 & 0.000 & 15.79 & 24 & 6 & 0 & R putamen \\
\hline & & & 0.003 & 4.99 & 0.000 & 12.32 & 32 & -6 & -2 & \\
\hline & & & 0.010 & 4.76 & 0.000 & 10.79 & 28 & -10 & 8 & \\
\hline \multirow[t]{3}{*}{0.000} & 1,151 & 0.000 & 0.005 & 4.90 & 0.000 & 11.73 & -26 & -2 & 4 & L putamen \\
\hline & & & 0.033 & 4.52 & 0.000 & 9.37 & -34 & -8 & 6 & \\
\hline & & & 0.056 & 4.41 & 0.000 & 8.77 & -16 & 22 & -6 & \\
\hline \multicolumn{11}{|c|}{ ET $>$ PD with template C } \\
\hline \multirow[t]{3}{*}{0.000} & 1,573 & 0.000 & 0.001 & 5.25 & 0.000 & 14.49 & 32 & -6 & -2 & R putamen \\
\hline & & & 0.001 & 5.23 & 0.000 & 14.34 & 22 & 6 & 2 & \\
\hline & & & 0.004 & 4.95 & 0.000 & 12.04 & 26 & 4 & 10 & \\
\hline \multirow[t]{3}{*}{0.000} & 1,115 & 0.000 & 0.017 & 4.66 & 0.000 & 10.17 & -26 & -2 & 2 & L putamen \\
\hline & & & 0.034 & 4.52 & 0.000 & 9.33 & -32 & -8 & 6 & \\
\hline & & & 0.047 & 4.44 & 0.000 & 8.97 & -22 & 18 & 4 & \\
\hline
\end{tabular}

residual squared difference between the images being normalized and the template (19), the contrast in the original image and the template must be similar. A previous study showed that the use of CBF instead of the ${ }^{18} \mathrm{~F}-\mathrm{FDG}$ template to normalize ${ }^{18} \mathrm{~F}$-FDG images resulted in different extents and peak heights of areas representing metabolic changes (20). Therefore, the use of generic perfusion templates is not adapted for ligands of the dopaminergic system, in which the specific tracer uptake is confined to the striatum with a very low cortical signal. Therefore, to optimize the normalization procedure it is necessary to design a specific template in which the spatial distribution of activity is similar to the one observed in the images to be normalized $(20,35)$. Our aim was to validate such an approach in a pilot SPECT study. An unresolved question is the minimal number of images required to obtain a reliable template. For example, the $\mathrm{CBF}\left(\mathrm{H}_{2}{ }^{15} \mathrm{O}\right)$ template provided within the SPM99 software was created with 12 normal scans. Most templates created for other brain tracers were obtained with a small number of subjects (i.e., 10-15 subjects) $(20,33,35,36)$. In our study, we used all of the validated normal images available in each center at the start of the research to create each template. Our results suggest that the difference in sample size of the subjects included for the 4 templates has a minimal impact on statistical outcomes.

Because of the limited number of subjects included, our study should be considered as exploratory. Specifically, the small sample size of the test population may mask true differences because of insufficient statistical power. However, all normalization procedures have been performed using the standard algorithms provided within the SPM software to permit the reproduction of our results.

We found that the normalization process was accurate even in pathologic images such as those of advanced PD patients. In addition, the normalization process has no major impact on striatal BR values, and the comparison between PD patients and controls is not affected by the warping of the images. To state this in another way, these results suggest that the location and magnitude of all 
TABLE 4

Comparison of SPM $t$ Maps $(P<0.001$ Uncorrected $)$ Obtained After Each Normalization

\begin{tabular}{|c|c|c|c|c|c|}
\hline \multirow[b]{2}{*}{ Template } & \multirow{2}{*}{$\begin{array}{l}\text { Cluster size } \\
\text { (voxels) }^{\star}\end{array}$} & \multicolumn{4}{|c|}{$\begin{array}{c}\text { Repartition of significant voxels } \\
\text { in striatum }(\%)^{\dagger}\end{array}$} \\
\hline & & $\mathrm{RPu}$ & $\mathrm{LPu}$ & $\mathrm{R} \mathrm{Cd}$ & $\mathrm{LCd}$ \\
\hline $\mathrm{A} 1$ & 2,489 & 32.1 & 23.5 & 7.6 & 3.9 \\
\hline A2 & 2,601 & 31.5 & 24.5 & 8.5 & 3.5 \\
\hline B & 2,619 & 29.6 & 23.9 & 10.2 & 5.2 \\
\hline C & 2,688 & 29.2 & 22.0 & 10.8 & 6.2 \\
\hline
\end{tabular}

*Total number of statistically different voxels between the 2 groups (6 PD patients < 6 ET patients).

${ }^{\dagger}$ Repartition in striatal subregions expressed in percentage of total number of significant voxels.

$\mathrm{R}=$ right; $\mathrm{Pu}=$ putamen; $\mathrm{L}=$ left; $\mathrm{Cd}=$ caudate nucleus.

significant changes between PD and ET subjects are well preserved by the normalization process performed using our template. Our analysis also suggests that a single template could normalize ${ }^{123}$ I-FP-CIT images acquired on different cameras and reconstructed using different procedures. Indeed, we show that the topography, the magnitude, and the statistical significance of the differences between PD and ET subjects are similar after normalizations to the different templates. Precisely, the magnitude of the decrease in BR values measured in the PD population with VOIs in the different normalized images was nearly similar, ranging from $41.5 \%$ to $43.9 \%$ in the right putamen and from $53.3 \%$ to $56.1 \%$ in the left putamen. Although there are some differences among the 4 templates, as suggested by Bland-Altman graphical analysis, these differences have no clinical impact on intergroup comparisons because of the strong correlations between values obtained after normalization to templates $\mathrm{A} 2, \mathrm{~B}$, and $\mathrm{C}$, and values obtained after normalization to template A1 (ICC > 0.98). Therefore, when a voxel-based SPM analysis was performed, the $t$ maps obtained by comparing PD with ET subjects were very similar from one template to another (Fig. 5). This extends the results obtained previously for ${ }^{18} \mathrm{~F}-\mathrm{FDG}$ and regional CBF images to ${ }^{123}$ I-FP-CIT images $(20,21)$. However, one should note that there are some similarities between the $\gamma$-cameras used in the different centers. Although we used different subjects and image reconstruction algorithms to create some variability between the templates, this work does not cover the wide range of acquisition and reconstruction protocols used in nuclear medicine departments specialized in brain SPECT investigation. However, this study design could be easily repeated in any center.

Eventually, another advantage of validating SPM spatial normalization is to offer the possibility of performing a fully automated VOI analysis of ${ }^{123}$ I-FP-CIT SPECT images. This was done by using a single template of predefined VOI obtained from the parcellation of the MNI single-subject MRI and applying these volumes to each spatially transformed parametric image. Therefore, one can use a unique 3D volume in all scans that might improve reproducibility. This step is crucial when assessing PD progression in follow-up studies.

\section{CONCLUSION}

This study suggests that an ${ }^{123}$ I-FP-CIT SPECT template can be constructed without any reference to MR images and provides accurate measurements of specific striatal activity. This normalization is reliable even with images obtained in PD patients who have a severe decrease in DAT uptake in the putamen. Although we included a limited number of subjects and $\gamma$-cameras that were partially similar, our results suggest that a single template can be used to normalize ${ }^{123}$ I-FP-CIT images obtained in different centers, without affecting the sensitivity and accuracy of the comparisons between small groups of patients.

\section{ACKNOWLEDGMENT}

We thank Dr. Edouard Duschesnay for help in this study.

\section{REFERENCES}

1. Kaufman MJ, Madras BK. Severe depletion of cocaine recognition sites associated with the dopamine transporter in Parkinson's-diseased striatum. Synapse. 1991;9:43-49.

2. Niznik HB, Fogel EF, Fassos FF, Seeman P. The dopamine transporter is absent in parkinsonian putamen and reduced in the caudate nucleus. $J$ Neurochem. 1991;56:192-198.

3. Guttman M, Burkholder J, Kish SJ, et al. $\left[{ }^{11} \mathrm{C}\right]$ RTI-32 PET studies of the dopamine transporter in early dopa-naive Parkinson's disease: implications for the symptomatic threshold. Neurology. 1997;48:1578-1583.

4. Winogrodzka A, Bergmans P, Booij J, van Royen EA, Janssen AG, Wolters EC. $\left[{ }^{123}\right.$ I]FP-CIT SPECT is a useful method to monitor the rate of dopaminergic degeneration in early-stage Parkinson's disease. J Neural Transm. 2001;108: 1011-1019.

5. Tissingh G, Booij J, Bergmans P, et al. Iodine-123-N-omega-fluoropropyl-2betacarbomethoxy-3beta-(4-iodophenyl)tropane SPECT in healthy controls and early-stage, drug-naive Parkinson's disease. J Nucl Med. 1998;39:1143-1148.

6. Benamer HT, Patterson J, Wyper DJ, Hadley DM, Macphee GJ, Grosset DG. Correlation of Parkinson's disease severity and duration with ${ }^{123}$ I-FP-CIT SPECT striatal uptake. Mov Disord. 2000;15:692-698.

7. Adams JR, van Netten H, Schulzer M, et al. PET in LRRK2 mutations: comparison to sporadic Parkinson's disease and evidence for presymptomatic compensation. Brain. 2005;128:2777-2785.

8. Booij J, Speelman JD, Horstink MW, Wolters EC. The clinical benefit of imaging striatal dopamine transporters with $\left[{ }^{123}\right.$ I]FP-CIT SPET in differentiating patients with presynaptic parkinsonism from those with other forms of parkinsonism. Eur J Nucl Med. 2001;28:266-272.

9. Chouker M, Tatsch K, Linke R, Pogarell O, Hahn K, Schwarz J. Striatal dopamine transporter binding in early to moderately advanced Parkinson's disease: monitoring of disease progression over 2 years. Nucl Med Commun. 2001;22:721-725.

10. Marek K, Innis R, van Dyck C, et al. $\left[{ }^{123} \mathrm{I}\right] \beta$-CIT SPECT imaging assessment of the rate of Parkinson's disease progression. Neurology. 2001;57:2089-2094.

11. Pirker W, Djamshidian S, Asenbaum S, et al. Progression of dopaminergic degeneration in Parkinson's disease and atypical parkinsonism: a longitudinal beta-CIT SPECT study. Mov Disord. 2002;17:45-53.

12. Parkinson Study Group. Dopamine transporter brain imaging to assess the effects of pramipexole vs levodopa on Parkinson disease progression. JAMA. 2002; 287(13):1653-1661.

13. Marek K, Jennings D, Seibyl J. Do dopamine agonists or levodopa modify Parkinson's disease progression? Eur J Neurol. 2002;9(suppl 3):15-22.

14. Laruelle M, Giddings SS, Zea-Ponce Y, et al. Methyl 3 beta-(4-[125I]iodophenyl)tropane-2 beta-carboxylate in vitro binding to dopamine and serotonin transporters under "physiological" conditions. J Neurochem. 1994;62:978-986. 
15. Abi-Dargham A, Gandelman MS, DeErausquin GA, et al. SPECT imaging of dopamine transporters in human brain with iodine-123-fluoroalkyl analogs of beta-CIT. J Nucl Med. 1996;37:1129-1133.

16. Friston KJ. Statistical parametric mapping. In: Thatcher RW, Hallett M, Zeffiro T, John ER, Huerta M, eds. Functional Neuroimaging. San Diego, CA: Academic Press; 1994: 79-93.

17. Friston KJ, Holmes AP, Worsley KJ, Poline J-P, Frith CD, Frackowiak RSJ. Statistical parametric maps in functional imaging: a general linear approach. Hum Brain Mapp. 1995;2:189-210.

18. Weeks RA, Cunningham VJ, Piccini P, Waters S, Harding AE, Brooks DJ. ${ }^{11} \mathrm{C}$ Diprenorphine binding in Huntington's disease: a comparison of region of interest analysis with statistical parametric mapping. J Cereb Blood Flow Metab. 1997; 17:943-949.

19. Ashburner J, Friston KJ. Nonlinear spatial normalization using basis functions. Hum Brain Mapp. 1999;7:254-266.

20. Gispert JD, Pascau J, Reig S, et al. Influence of the normalization template on the outcome of statistical parametric mapping of PET scans. Neuroimage. 2003;19:601-612.

21. Ishii K, Willoch F, Minoshima S, et al. Statistical brain mapping of ${ }^{18}$ F-FDG PET in Alzheimer's disease: validation of anatomic standardization for atrophied brains. J Nucl Med. 2001;42:548-557.

22. Brett M, Leff AP, Rorden C, Ashburner J. Spatial normalization of brain images with focal lesions using cost function masking. Neuroimage. 2001;14:486-500.

23. Hughes AJ, Daniel SE, Kilford L, Lees AJ. Accuracy of clinical diagnosis of idiopathic Parkinson's disease: a clinico-pathological study of 100 cases. J Neurol Neurosurg Psychiatry. 1992;55:181-184.

24. Defer GL, Widner H, Marie RM, Remy P, Levivier M. Core assessment program for surgical interventional therapies in Parkinson's disease (CAPSIT-PD). Mov Disord. 1999;14:572-584.

25. Booij J, Hemelaar TG, Speelman JD, de Bruin K, Janssen AG, van Royen EA. One-day protocol for imaging of the nigrostriatal dopaminergic pathway in Parkinson's disease by [123I]FPCIT SPECT. J Nucl Med. 1999;40:753-761.
26. Bonab AA, Fischman AJ, Alpert NM. Comparison of 4 methods for quantification of dopamine transporters by SPECT with [ ${ }^{123}$ I]IACFT. J Nucl Med. 2000; 41:1086-1092.

27. El Fakhri G, Maksud P, Kijewski MF, et al. Scatter and cross-talk corrections in simultaneous Tc-99m/I-123: brain SPECT using constrained factor analysis and artificial neural networks. IEEE Trans Nucl Sci. 2000;47:1573-1580.

28. Veltman D, Hutton C. SPM99 Manual. Technical report, Wellcome Department of Imaging Neuroscience, University College London. url: www.fil.ion.ucl.ac.uk/ spm/doc/manual/, 2001. Accessed August 3, 2007.

29. Plotkin M, Amthauer H, Klaffke S, et al. Combined ${ }^{123}$ I-FP-CIT and ${ }^{123}$ I-IBZM SPECT for the diagnosis of parkinsonian syndromes: study on 72 patients. J Neural Transm. 2005;112:677-692.

30. Bland JM, Altman DG. Measurement error and correlation coefficients. BMJ. 1996;313(7048):41-42.

31. Bland JM, Altman DG. Statistical methods for assessing agreement between two methods of clinical measurement. Lancet. 1986;1(8476):307-310.

32. Tzourio-Mazoyer N, Landeau B, Papathanassiou D, et al. Automated anatomical labeling of activations in SPM using a macroscopic anatomical parcellation of the MNI MRI single-subject brain. Neuroimage. 2002;15:273-289.

33. Meyer JH, Gunn RN, Myers R, Grasby PM. Assessment of spatial normalization of PET ligand images using ligand-specific templates. Neuroimage. 1999;9:545-553.

34. Ma Y, Dhawan V, Mentis M, Chaly T, Spetsieris PG, Eidelberg D. Parametric mapping of $\left[{ }^{18} \mathrm{~F}\right] \mathrm{FPCIT}$ binding in early stage Parkinson's disease: a PET study. Synapse. 2002;45:125-133.

35. Signorini M, Paulesu E, Friston K, et al. Rapid assessment of regional cerebral metabolic abnormalities in single subjects with quantitative and nonquantitative $\left[{ }^{18}\right.$ F]FDG PET: a clinical validation of statistical parametric mapping. Neuroimage. 1999;9:63-80.

36. Nagano-Saito A, Kato T, Arahata Y, et al. Cognitive- and motor-related regions in Parkinson's disease: FDOPA and FDG PET studies. Neuroimage. 2004;22: $553-561$. 